\title{
The Effect of Oral Administration of Black Sticky Rice with Giant Embryo on Brain GABA Concentrations
}

\author{
Woo-Young Jung ${ }^{1}$, Sung-Gon Kim ${ }^{1,2} \bowtie$, Hyeon-Kyeong Kim³ ${ }^{3}$, Sung-Young Huh', Dae-Wook Kim¹, \\ Dong-Uk Yoon ${ }^{1}$, Chae Ha Yang ${ }^{4}$, Hee Young Kim4, and Eun Young Jang ${ }^{4,5}$ \\ ${ }^{1}$ Department of Psychiatry, Pusan National University Yangsan Hospital, Yangsan, Republic of Korea \\ 2Department of Psychiatry, Pusan National University School of Medicine, Yangsan, Republic of Korea \\ ${ }^{3}$ Medical Research Institute, Pusan National University, Busan, Republic of Korea \\ ${ }^{4}$ Department of Physiology, College of Korean Medicine, Daegu Haany University, Daegu, Republic of Korea \\ ${ }^{5}$ Research Center for Convergence Toxicology, Korea Institute of Toxicology, Daegeon, Republic of Korea
}

Objective Black sticky rice with giant embryo (BSRGE) contains high GABA content and affects alcohol-related indices among social drinkers, and alcohol intake and anxiety-related behavior of mice. However, it is unknown whether the intake of BSRGE affects GABAergic activity of brain directly. The purpose of this study is to elucidate the effect of oral administration of BSRGE on brain GABA concentrations compared with commercially available GABA compound and regular feeds.

Methods Twenty-one male C57BL/6 mice were assigned to BSRGE, a regular feed (AIN-76) lacking GABA, and a regular feed containing GABA compound. After feeding freely for $48 \mathrm{~h}$, the cortex and striatum were separated from the brain. An enzyme-linked immunosorbent assay was conducted to measure GABA and glutamate concentrations in mouse brain.

Results The GABA concentration of the BSRGE group was higher than that of regular feed and GABA compound group ( $<<0.001$ ). However, the GABA compound group showed no significant difference from the regular feed group ( $\mathrm{p}=0.50$ ).

Conclusion Intake of BSRGE containing high GABA content increased GABA concentrations in mouse brain compared with regular feed unlike GABA compound. The results of this study constitute an important basis for further investigations into the clinical applications of BSRGE.

Psychiatry Investig 2019;16(8):615-620

Key Words BSRGE, ELISA, GABA, Glutamate.

\section{INTRODUCTION}

Gamma-aminobutyric acid (GABA) is the most widely distributed inhibitory neurotransmitter in the central nervous system (CNS). ${ }^{1}$ Excessive GABAergic signaling is associated with sedation, amnesia, and ataxia. Conversely, the mildest attenuation of GABAergic signaling induces arousal, anxiety, restlessness, insomnia, and exaggerated reactivity. ${ }^{2}$ Instability of GABA function in the CNS has been linked to psychiatric disorders. ${ }^{3}$ For example, GABA receptors are clearly involved in the acute effects of alcohol, tolerance, self-administration

\footnotetext{
Received: March 27, 2019 Accepted: May 13, 2019

$\triangle$ Correspondence: Sung-Gon Kim, MD, PhD

Department of Psychiatry, Pusan National University School of Medicine, Mulgeum-eup, Yangsan 50612, Republic of Korea

Tel: +82-55-360-2123, Fax: +82-55-360-2153, E-mail: sungkim@pusan.ac.kr

(c) This is an Open Access article distributed under the terms of the Creative Commons Attribution Non-Commercial License (https://creativecommons.org/licenses/bync/4.0) which permits unrestricted non-commercial use, distribution, and reproduction in any medium, provided the original work is properly cited.
}

and alcohol dependence. ${ }^{4}$ In addition, dysfunction of the central GABA system is related to anxiety and depressive disorder. ${ }^{5}$ Therefore, specific medications related to GABA have been prescribed to manage the psychiatric and neurological disorders. ${ }^{5}$ Acamprosate used to treat alcohol use disorder contains a chemical structure similar to that of $\mathrm{GABA}^{6}$ and has been found to modulate NMDA and GABA transmission. ${ }^{7}$ In both human and animal studies, positive modulators of GABA receptors generally show anxiolytic effect, while negative modulators induce anxiety. ${ }^{5}$ GABA agonists, GABA analogs, and agents affecting transmitter metabolism such as benzodiazepine, gabapentin, and valproate showed anti-anxiety, sedative and anti-epileptic activity. ${ }^{5,8,9}$

Meanwhile, the rice breeding team of the National Institute of Crop Science (NICS) in Korea developed the Black Sticky Rice with Giant Embryo (BSRGE, "Nunkeunheugchal," registration No. 01-0001-2014-4), which is created in 2012 with the aim of maximizing the functional ingredients of rice. The 
embryonal weight of "Nunkeunheugchal" is $1.46 \mathrm{mg}$. It is 3 times heavier than the general rice "Josaengheugchal." The embryo contains a GABA concentration of $34 \mathrm{mg} / 100 \mathrm{~g}$, which is 7 times higher than in "Josaengheugchal." The GABA content of the germinated rice in "Nunkeunheugchal" is $88 \mathrm{mg} / 100$ g, which is 1.7 times higher than that of "Josaengheugchal"10 (Table 1). Investigations suggest an association between alcohol intake, alcohol-related indices and effects of BSRGE. Kim et al. ${ }^{11}$ reported that feeding BSRGE freely to C57BL/6 mice induced a significant decline in 2-h alcohol intake compared with the control group. The changes in 2-h alcohol intake were attributed to higher GABA levels in BSRGE than in the general brown rice. Jung et al. ${ }^{12}$ reported that alcohol in combination with GABA extract in social drinkers affected alcohol-related indices after binge drinking associated with gender-related differences in sedation. Based on the results of these two studies, BSRGE containing high GABA concentrations might suggest an association with alcohol-related effects and alcohol intake. Additionally, the BSRGE intake of C57/BL6 mice reduced anxiety-related behavior in elevated plus maze test. ${ }^{13}$

The results of these previous studies suggest that the daily intake of BSRGE may alter the degree of alcohol intake, alcohol-related indices and anxiety-related behaviors, suggesting the need for comprehensive clinical investigations. In recent years GABA has been used as a food supplement. In the US and Europe, GABA is considered as a "food constituent" and a "dietary supplement," respectively. ${ }^{14} \mathrm{GABA}$-enriched foods including many cereals, dairy products, meat, vegetables and beverages were already reported. ${ }^{15}$ Interestingly, GABA has long been considered not to cross the blood brain barrier (BBB), which prompted questions about its pharmacological mechanisms underlying its beneficial effects. ${ }^{14}$ Because BBB is a tightly sealed layer of cerebral endothelial cells and prevents the entry of most solutes from the brain on the basis of size, charge and lipid solubility, especially polar or hydrophilic molecules that are $>500 \mathrm{Da}$, which cannot cross the tight junction. ${ }^{16}$ However, GABA-enriched foods and supplements have been shown to relieve anxiety and induce better sleep quality, in addition to other beneficial effects. ${ }^{14}$ In addition, Yoto et al. ${ }^{17}$ reported that oral intake of GABA affects mood and CNS activities under mental stress induced by mental tasks. A few studies suggested that the BBB is far more dynamic than presumed in the past, and limited solute diffusion occurred via transcytosis, carrier-mediated transport, or simple diffusion of hydrophobic substances. ${ }^{18}$ Thus, the role of oral GABA diffusion across $\mathrm{BBB}$ directly, and its effect on GABA receptors in the brain is disputed. However, it is difficult to identify the mechanisms underlying the BBB permeability of GABA or its effect in the human brain.

Therefore, the purpose of the present study is to elucidate the effect of oral administration of Black Sticky Rice with Giant Embryo (BSRGE) on brain GABA concentration using ELISA, compared with administration of single GABA compound and controlled regular feed. In addition, change of glutamate concentration in the brain is also analyzed because of overlapping pathways between brain GABA and glutamate metabolism and importance of glutamate/GABA balance or excitatory/inhibitory balance in CNS synaptic function. ${ }^{19}$

\section{METHODS}

\section{Study animals}

Twenty-one 8-week-old male C57BL/6 mice were used in the present study. A temperature of $23 \pm 2^{\circ} \mathrm{C}$ and a humidity of $55-65 \%$ were maintained, and feed and water were provided ad libitum. After acclimation of the mice for 7 days, the study was performed. The present study was approved by the Pusan National University Institutional Animal Care and Use Committee (PNU-2015-0777).

\section{Reagents and materials}

The glutamate and GABA ELISA kits used were manufactured by Novatein Bioscience (Boston, MA, USA) and MyBioScience (San Diego, CA, USA) respectively. Other materials were obtained from Sigma Aldrich (San Diego, CA, USA).

\section{Distribution of experimental group and feed administration (Table 2)}

Experimental animals were divided into groups of 7 each and assigned to BSRGE, regular feed, and GABA compound (Sigma Aldrich) combined with regular feed. Regular feed served as the control feed. The commercially available GABA compound was also provided to compare with BSRGE enriched with GABA. The regular feed lacked GABA and glutamate content. The groups treated with BSRGE and GABA

Table 1. Physicochemical property of 'Nunkeunheugchal'

\begin{tabular}{|c|c|c|c|c|c|c|c|c|c|}
\hline \multirow[b]{2}{*}{ Variety } & \multirow{2}{*}{$\begin{array}{l}\text { Single embryo } \\
\text { weight (mg) }\end{array}$} & \multicolumn{2}{|c|}{ GABA (mg/100 g) } & \multirow{2}{*}{$\begin{array}{l}\text { Anthocyanin } \\
(\mathrm{mg} / 100 \mathrm{~g})\end{array}$} & \multirow{2}{*}{$\begin{array}{l}\gamma \text {-Oryzanol } \\
(\mathrm{mg} / 100 \mathrm{~g})\end{array}$} & \multirow{2}{*}{$\begin{array}{l}\text { Lipid } \\
(\%)\end{array}$} & \multicolumn{3}{|c|}{ Minerals (mg/100 g) } \\
\hline & & Brown rice & $\begin{array}{l}\text { Germinated } \\
\text { brown rice }\end{array}$ & & & & $\mathrm{Ca}$ & $\mathrm{Mg}$ & $\mathrm{Fe}$ \\
\hline Nunkeunheugchal & 1.46 & 34 & 88 & 129 & 32 & 2.6 & 33.8 & 136.6 & 4.57 \\
\hline Josaengheugchal & 0.51 & 5 & 55 & 118 & 29 & 3.1 & 23.5 & 119.3 & 2.07 \\
\hline
\end{tabular}


compound with regular feed were ensured exposure to similar GABA content. Each group was permitted free intake for 48 h. The body weight and food levels were determined before and after feeding freely for $48 \mathrm{~h}$.

\section{Measurement of GABA and glutamate concentrations in mouse brain}

ELISA was used to measure GABA and glutamate concentrations in the mouse brain. The animals were exposed to isoflurane anesthesia and their cerebral cortex and striatum were separated. The brain tissue was pulverized in artificial cerebrospinal fluid (125 mM NaCl, $2.5 \mathrm{mM} \mathrm{KCl}, 1.3 \mathrm{mM} \mathrm{MgSO} 4$, $26 \mathrm{mM} \mathrm{NaHCO} 3,1.25 \mathrm{mM} \mathrm{NaH}_{2} \mathrm{PO}$, $2.0 \mathrm{mM} \mathrm{CaCl}_{2}$, and $10 \mathrm{mM}$ glucose, $\mathrm{pH}-7.4$ ), followed by centrifugation under $4^{\circ} \mathrm{C}$ for $15 \mathrm{~min}$. After 100 -fold dilution, the optical density of glutamate and GABA was measured at $450 \mathrm{~nm}$ by ELISA according to the kit protocol. The required concentrations of GABA and glutamate were calculated from the graph plotted using standard and optical density values obtained.

Table 2. Composition of experimental feeds*

\begin{tabular}{lccc}
\hline \multicolumn{1}{c}{ Ingredient } & Regular feed & $\begin{array}{c}\text { GABA compound } \\
\text { with regular feed }\end{array}$ & BSRGE \\
\hline Casein & 20.0 & 20.0 & 20.0 \\
Sucrose & 50.0 & 49.9 & 10.0 \\
Cornstarch & 15.0 & 15.0 & 15.0 \\
Corn oil & 5.0 & 5.0 & 5.0 \\
Cellulose & 5.0 & 5.0 & 5.0 \\
Mineral mixture & 3.5 & 3.5 & 3.5 \\
Vitamin mixture & 1.0 & 1.0 & 1.0 \\
Choline bitartrate & 0.2 & 0.2 & 0.2 \\
DL-methionine & 0.3 & 0.3 & 0.3 \\
Rice bran in embryo ${ }^{\dagger}$ & - & - & 40.0 \\
GABA $^{\ddagger}$ & & 0.1 & \\
Total & 100.0 & 100.0 & 100.0 \\
\hline
\end{tabular}

*all components are expressed in units of g/100 g feeds, ${ }^{\dagger} \mathrm{GABA}$ content of rice bran in embryo is $190 \mathrm{mg} / 100 \mathrm{~g}$, ‡GABA: sigma. BSRGE: Black Sticky Rice Giant Embryo

\section{Statistical analysis}

The mean and standard deviations of changes in both body weight and food intake per body weight were calculated using descriptive statistics, and the statistical significance was analyzed by one-way ANOVA. The homogeneity of variance was tested and post hoc analysis was performed by Bonferroni and Tamhane method. The mean and standard deviation of optical density measured by ELISA was also confirmed by descriptive statistics. The homogeneity of variance was tested and post hoc analysis was performed by the Tukey method. SPSS (ver. 18; SPSS Inc., Chicago, IL, USA), a social science statistics program for Windows, was used for statistical analysis and statistical significance level was defined as $\mathrm{p}<0.05$.

\section{RESULTS}

\section{Changes in mouse weight and amount of feed intake} (Table 3)

The 48-h changes in body weight were: $-1.70 \pm 0.68$ (g) in the BSRGE group, $-0.89 \pm 0.51(\mathrm{~g})$ in the regular feed, and -0.22 \pm 0.25 (g) in the GABA compound group, with statistically significant differences $(\mathrm{p}<0.001)$. Post hoc analysis showed a greater weight loss in the BSRGE group compared with the GABA compound group ( $\mathrm{p}=0.002$ ). The regular feed group showed significantly higher weight loss than the GABA compound group ( $\mathrm{p}=0.038)$.

The amount of food intake over a period of $48 \mathrm{~h}$ was 179.38 $\pm 41.60 \mathrm{~g} / \mathrm{kg}$ in the BSRGE group, $205.47 \pm 27.59 \mathrm{~g} / \mathrm{kg}$ in the regular feed group and $238.48 \pm 21.16 \mathrm{~g} / \mathrm{kg}$ in the group treated with GABA compound, with significant differences ( $\mathrm{p}=0.009)$. Post hoc analysis showed that the amount of feed intake in BSRGE group was significantly less than in GABA compound group $(\mathrm{p}=0.007)$.

\section{Altered GABA and glutamate concentrations after each 48-h food intake (Table 4)}

\section{GABA concentrations of striatum and cortex (BSRGE vs. regular feed and GABA compound)}

The GABA concentration of striatum and cortex after a 48-h feeding was $5.72 \pm 0.22(\mu \mathrm{mol} / \mathrm{L})$ in the BSRGE group,

Table 3. Amount of each feed intake and 48-h changes in weight

\begin{tabular}{|c|c|c|c|c|c|c|c|}
\hline & $\begin{array}{l}\text { BSRGE } \\
\text { (a) }\end{array}$ & $\begin{array}{l}\text { Regular feed } \\
\text { (b) }\end{array}$ & $\begin{array}{c}\text { GABA compound } \\
\text { with regular feed }(\mathrm{C})\end{array}$ & $\begin{array}{l}\mathrm{p} \text { value } \\
(\mathrm{a}, \mathrm{b}, \mathrm{c})\end{array}$ & $\begin{array}{l}\text { p value } \\
\text { (a vs. b) }\end{array}$ & $\begin{array}{l}\text { p value } \\
\text { (a vs. c) }\end{array}$ & $\begin{array}{l}\text { p value } \\
\text { (b vs. c) }\end{array}$ \\
\hline $\begin{array}{l}\text { Amount of intake }(\mathrm{g} / \mathrm{kg}) \\
(\text { mean } \pm \mathrm{SD})\end{array}$ & $179.38 \pm 41.60$ & $205.47 \pm 27.59$ & $238.48 \pm 21.16$ & $0.009^{*}$ & 0.409 & $0.007^{*}$ & 0.192 \\
\hline $\begin{array}{l}\text { Change of weight }(\mathrm{g}) \\
(\text { mean } \pm \mathrm{SD})\end{array}$ & $-1.70 \pm 0.68$ & $-0.89 \pm 0.51$ & $-0.22 \pm 0.25$ & $<0.001^{*}$ & 0.082 & $0.002^{*}$ & $0.038^{*}$ \\
\hline
\end{tabular}


Table 4. Effect of GABA and glutamate concentrations on cortex and striatum of mouse after 48-h administration each feed

\begin{tabular}{|c|c|c|c|c|c|c|c|}
\hline & $\begin{array}{l}\text { BSRGE } \\
\text { (a) }\end{array}$ & $\begin{array}{l}\text { Regular feed } \\
\text { (b) }\end{array}$ & $\begin{array}{l}\text { GABA compound } \\
\text { (c) }\end{array}$ & $\begin{array}{l}\mathrm{p} \text { value } \\
(\mathrm{a}, \mathrm{b}, \mathrm{c})\end{array}$ & $\begin{array}{l}\text { p value } \\
\text { (a vs. b) }\end{array}$ & $\begin{array}{l}\text { p value } \\
\text { (a vs. c) }\end{array}$ & $\begin{array}{l}\text { p value } \\
\text { (b vs. c) }\end{array}$ \\
\hline $\begin{array}{l}\text { Brain GABA concentration } \\
(\text { mean } \pm \text { SEM })(\mu \mathrm{mol} / \mathrm{L})\end{array}$ & $5.72 \pm 0.22$ & $4.53 \pm 0.08$ & $4.31 \pm 0.05$ & $<0.001^{*}$ & $<0.001^{*}$ & $<0.001^{*}$ & 0.496 \\
\hline $\begin{array}{l}\text { Brain glutamate concentration } \\
(\text { mean } \pm \text { SEM })(\mu \mathrm{mol} / \mathrm{L})\end{array}$ & $0.41 \pm 0.02$ & $0.44 \pm 0.03$ & $0.32 \pm 0.01$ & $0.005^{*}$ & 0.674 & $0.030^{*}$ & $0.005^{*}$ \\
\hline
\end{tabular}

${ }^{*} \mathrm{p}<0.05$. BSRGE: Black Sticky Rice Giant Embryo

$4.53 \pm 0.08(\mu \mathrm{mol} / \mathrm{L})$ in the regular feed group, and $4.31 \pm 0.05$ $(\mu \mathrm{mol} / \mathrm{L})$ in the GABA compound group, with statistically significantly differences $(\mathrm{p}<0.001)$. Post hoc analysis revealed higher GABA concentration in the BSRGE group compared with the regular feed group $(\mathrm{p}<0.001)$ and GABA compound group $(\mathrm{p}<0.001)$.

\section{Glutamate concentrations of striatum and cortex (BSRGE vs. GABA compound)}

The glutamate concentration of striatum and cortex after a 48-h feeding was $0.41 \pm 0.02(\mu \mathrm{mol} / \mathrm{L})$ in the BSRGE group, $0.44 \pm 0.03(\mu \mathrm{mol} / \mathrm{L})$ in the regular feed group and $0.32 \pm 0.01$ $(\mu \mathrm{mol} / \mathrm{L})$ in the GABA compound group, with statistically significantly differences $(\mathrm{p}=0.005)$. Post hoc analysis revealed higher glutamate concentration in the BSRGE group compared with that of GABA compound group $(\mathrm{p}=0.03)$. The glutamate concentration of the groups exposed to regular feed was higher than in the GABA compound group ( $p=0.005)$.

\section{DISCUSSION}

In the present study, the oral intake of BSRGE significantly increased GABA concentration in mouse brain compared with exposure to GABA compound and regular feed. The glutamate concentration of mouse brain after BSRGE oral intake showed no significant changes compared with regular feed. Accordingly, the oral intake of BSRGE appears to influence the brain GABA concentrations in mouse.

Initial studies from the fifties reported the inability of GABA to penetrate the $\mathrm{BBB}$, which was further corroborated by several studies. ${ }^{14}$ However, GABA permeability into the brain through the BBB is still disputed, because several studies reported that GABA diffuses across the BBB, in small amounts. ${ }^{14,18}$ Boonstra et al. ${ }^{14}$ proposed that this inconsistency could be attributed to variation in chemical compounds, used in studies reporting the permeability of GABA, methods of administration (oral versus intraperitoneal), and the species used such as rats, mice, rabbits and dogs. Previous reports demonstrated minimal blood-to-brain transfer of GABA under normal physiological conditions. The BBB efflux of GABA exceeds its BBB influx by 16 -fold. ${ }^{20}$ However, several previous studies suggested that the permeability of GABA may be altered under certain conditions. For example, Shyamaladevi et al. ${ }^{18}$ reported that GABA levels increased in brain following the administration of higher GABA and L-arginine concentrations at $10 \mathrm{~min}$. They suggested that GABA penetrated into brain under conditions of increased brain nitric oxide concentration, and L-arginine increases BBB permeability to GABA. In addition, the synthesis of GABA carriers, which penetrated the $\mathrm{BBB}$ and were metabolized to GABA inside the CNS were also investigated. Frey et al. ${ }^{21}$ reported that the convulsant thresholds were increased after oral administration of cetyl GABA. Jacob et al. ${ }^{22}$ reported that intraperitoneal injection of di-GABA lipid ester produced a considerable inhibition of general motor activity in mice. Meanwhile, Biswas et al. ${ }^{23}$ reported that high doses of intraperitoneal GABA injection altered the monoamine levels and their synthesis, similar to levels observed after intra-cerebroventricular injection. They suggested that a limited diffusion of GABA from blood into brain induced physiological changes in monoaminergic neurons. Thus, although the results of this study cannot conclusively determine whether BSRGE GABA component can pass through $\mathrm{BBB}$, daily intake of BSRGE in previous studies altered the degree of alcohol intake, ${ }^{11}$ alcohol-related indices ${ }^{12}$ and anxiety-related behaviors ${ }^{13}$ and its results may be related to an increase of GABA concentration and neurotransmission in CNS after BSRGE intake.

The glutamate concentration of BSRGE group was not different from that of the regular feeding group. This result is not surprising because of the constant regulation of brain glutamate concentrations. Glutamate is typically considered as the most important excitatory neurotransmitter in CNS. However, prolonged elevation in glutamate levels in the brain interstitial fluid (ISF) are highly cytotoxic, a phenomenon known as excitotoxicity. ${ }^{24}$ The overexcitation of neurons by glutamate may result in calcium influx that activates catabolic enzymes, resulting in neuronal death. Therefore, glutamate normally does not enter the brain. Additional reports indicate that it may be actively extruded from brain. ${ }^{25}$ The factors underlying the significantly lower glutamate concentration following exposure to GABA compound compared with regular feed are unclear. Glutamate is extensively metabolized via glutamine syn- 
thetase to glutamine or via aminotransferases or glutamate dehydrogenase to alpha-ketoglutarate. Conversely, glutamate can be synthesized from glutamine and alpha-ketoglutarate ${ }^{24}$ and glutamate also serves as the precursor for GABA synthesis, catalyzed by glutamate decarboxylase ${ }^{26}$ Further studies are needed to determine whether oral administration of GABA compound affected its metabolism.

The results of food intake and weight loss in this study cannot be established firmly, because they were not the primary outcomes of the study although it is interesting results. The BSRGE group consumed less food and showed greater weight loss compared with the GABA compound group. Interestingly, GABA and glutamate are known to be related to appetite regulation and feeding behavior. The hypothalamus is the principal appetite control site of brain and contains several peripheral signals that modulate the activity of orexigenic and anorexigenic neurons, including the orexigenic peptide ghrelin and the anorexigenic hormones insulin and leptin. Glutamate and GABA are the two neurotransmitters that account for most of the synaptic activity in the hypothalamus. ${ }^{27}$ In addition, GABA has been suggested to play an important role in the recognition of the type, quantity and quality of food by regulating the signal transmission between neurons in brain circuits. ${ }^{28}$ Hypoglycemic periods hinder GABA release in the substantia nigra and the ventral tegmental area, which are areas that project to the frontal cortex, and raise dopamine concentration and disinhibition of the frontal cortex. ${ }^{29}$ The prefrontal cortex plays a role in cognitive functions such as food selection. ${ }^{30}$

This study has several limitations. First, a small number of experimental mice were used in this study. Second, a significantly longer duration of feeding BSRGE is needed to observe the maintenance of GABA and glutamate concentrations in CNS. Third, only the striatum and cortex, and not the whole brain or additional area of brain, were used to measure GABA and glutamate concentrations in the CNS. The striatum and cortex in the brain contain an abundance of GABA receptors. ${ }^{31}$ Moreover, because striatum mediates the reward process, motor control, executive cognition and social behavior, ${ }^{32}$ it may represent a valuable area of investigation in the study. Despite these limitations, this study demonstrated that the daily intake of rice enriched with GABA might influence the GABA concentrations in CNS. Therefore, this study can be considered as a preliminary study in our investigation into the clinical effects of GABA extract.

\section{Acknowledgments}

This study was supported by Research institute for Convergence of biomedical science and technology (30-2016-009), Pusan National University Yangsan Hospital.

\section{Conflicts of Interest}

The authors have no potential conflicts of interest to disclose.

\section{Author Contributions}

Conceptualization: Sung-Gon Kim, Hyeon-Kyeong Kim. Data curation: Sung-Gon Kim, Chae Ha Yang, Hee Young Kim, Eun Young Jang. Formal analysis: Woo-Young Jung, Sung-Gon Kim, Hyeon-Kyeong Kim, Chae Ha Yang, Hee Young Kim, Eun Young Jang. Funding acquisition: Sung-Gon Kim, Hyeon-Kyeong Kim. Investigation: Sung-Gon Kim, Hyeon-Kyeong Kim. Methodology: Sung-Gon Kim, Hyeon-Kyeong Kim, Sung-Young Huh, Dae-Wook Kim, Dong-Uk Yoon, Chae Ha Yang, Hee Young Kim, Eun Young Jang. Project administration: Woo-Young Jung, Sung-Gon Kim, Hyeon-Kyeong Kim, Sung-Young Huh, Dae-Wook Kim, Dong-Uk Yoon, Chae Ha Yang, Hee Young Kim, Eun Young Jang. Resources: SungGon Kim, Hyeon-Kyeong Kim. Software: Sung-Gon Kim, Hyeon-Kyeong Kim, Chae Ha Yang, Hee Young Kim, Eun Young Jang. Supervision: SungGon Kim. Validation: Woo-Young Jung, Sung-Gon Kim, Hyeon-Kyeong Kim, Chae Ha Yang, Hee Young Kim, Eun Young Jang. Visualization: Sung-Gon Kim, Hyeon-Kyeong Kim. Writing_original draft: Woo-Young Jung, Sung-Gon Kim.

\section{ORCID iDs}

Sung-Gon, Kim https://orcid.org/0000-0001-7566-941X

Woo-Young Jung https://orcid.org/0000-0001-5844-7890

\section{REFERENCES}

1. Bormann J. The 'ABC' of GABA receptors. Trends Pharmacol Sci 2000;21:16-19.

2. Nutt D. GABAA receptors: subtypes, regional distribution, and function. J Clin Sleep Med 2006;2:S7-S11.

3. Al-Sarraf H. Transport of 14C-gamma-aminobutyric acid into brain, cerebrospinal fluid and choroid plexus in neonatal and adult rats. Brain Res Dev Brain Res 2002;139:121-129.

4. Grobin AC, Matthews DB, Devaud LL, Morrow AL. The role of GABA(A) receptors in the acute and chronic effects of ethanol. Psychopharmacology (Berl) 1998;139:2-19.

5. Kalueff AV, Nutt DJ. Role of GABA in anxiety and depression. Depress Anxiety 2007;24:495-517.

6. Mason BJ, Heyser CJ. The neurobiology, clinical efficacy and safety of acamprosate in the treatment of alcohol dependence. Expert Opin Drug Saf 2010;9:177-188.

7. Kalk NJ, Lingford-Hughes AR. The clinical pharmacology of acamprosate. Br J Clin Pharmacol 2014;77:315-323.

8. Goa KL, Sorkin EM. Gabapentin. A review of its pharmacological properties and clinical potential in epilepsy. Drugs 1993;46:409-427.

9. Sandford JJ, Argyropoulos SV, Nutt DJ. The psychobiology of anxiolytic drugs. Part 1: Basic neurobiology. Pharmacol Ther 2000;88:197-212.

10. Park DS, Hwang UH, Park SK, Lee JH, Han SI, Cho JY, et al. A waxy black giant embryo early maturing rice variety 'Nunkenheugchal'. Korean J Breed Sci 2015;47:68-74.

11. Kim HK, Kim SG, Lee JS, Lee SS, Jung WY, Han SI. Effect of feeding with high c-aminobutyric acid (GABA) containing giant embryo black sticky rice (Oryza sativa L.) on alcohol intake in C57BL/6 mice. J Life Sci 2013;23:698-702.

12. Jung WY, Kim SG, Kim HK, Lee JS, Han SI, Choe S, et al. Effect of GABA extract of black sticky rice with giant embryo on alcohol-related indices after acute alcohol intake in social drinkers. Alcohol Clin Exp Res 2015;39:1212-1218.

13. Jung WY, Kim SG, Lee JS, Kim HK, Son BG, Kim JW, et al. Effect of feeding high gamma-aminobutyric acid-containing giant embryo black sticky rice (Oryza sativa L.) on anxiety-related behavior of C57BL/6 mice. J Med Food 2017;20:777-781.

14. Boonstra E, de Kleijn R, Colzato LS, Alkemade A, Forstmann BU, 
Nieuwenhuis S. Neurotransmitters as food supplements: the effects of GABA on brain and behavior. Front Psychol 2015;6:1520.

15. Diana M, Quilez J, Rafecas M. Gamm-aminobutyric acid as a bioactive compound in foods:a review. J Funct Foods 2014:407-420.

16. Brightman MW, Reese TS. Junctions between intimately apposed cell membranes in the vertebrate brain. J Cell Biol 1969;40:648-677.

17. Yoto A, Murao S, Motoki M, Yokoyama Y, Horie N, Takeshima K, et al. Oral intake of gamma-aminobutyric acid affects mood and activities of central nervous system during stressed condition induced by mental tasks. Amino Acids 2012;43:1331-1337.

18. Shyamaladevi N, Jayakumar AR, Sujatha R, Paul V, Subramanian EH. Evidence that nitric oxide production increases gamma-amino butyric acid permeability of blood-brain barrier. Brain Res Bull 2002;57:231236.

19. El-Ansary A, Al-Ayadhi L. GABAergic/glutamatergic imbalance relative to excessive neuroinflammation in autism spectrum disorders. J Neuroinflammation 2014;11:189.

20. Kakee A, Takanaga H, Terasaki T, Naito M, Tsuruo T, Sugiyama Y. Efflux of a suppressive neurotransmitter, GABA, across the blood-brain barrier. J Neurochem 2001;79:110-118.

21. Frey HH, Loscher W. Cetyl GABA: effect on convulsant thresholds in mice and acute toxicity. Neuropharmacology 1980;19:217-220.

22. Jacob JN, Hesse GW, Shashoua VE. Gamma-Aminobutyric acid esters. 3. Synthesis, brain uptake, and pharmacological properties of C-18 glyceryl lipid esters of GABA with varying degree of unsaturation. J Med Chem 1987;30:1573-1576.
23. Biswas B, Carlsson A. The effect of intraperitoneally administered GABA on brain monoamine metabolism. Naunyn Schmiedebergs Arch Pharmacol 1977;299:47-51.

24. Cederberg HH, Uhd NC, Brodin B. Glutamate efflux at the bloodbrain barrier: cellular mechanisms and potential clinical relevance. Arch Med Res 2014;45:639-645.

25. Lee WJ, Hawkins RA, Vina JR, Peterson DR. Glutamine transport by the blood-brain barrier: a possible mechanism for nitrogen removal. Am J Physiol 1998;274:C1101-1107.

26. Battaglioli G, Liu H, Martin DL. Kinetic differences between the isoforms of glutamate decarboxylase: implications for the regulation of GABA synthesis. J Neurochem 2003;86:879-887.

27. Delgado TC. Glutamate and GABA in Appetite Regulation. Front Endocrinol (Lausanne) 2013;4:103.

28. Karra E, Chandarana K, Batterham RL. The role of peptide YY in appetite regulation and obesity. J Physiol 2009;587:19-25.

29. Geary N. Pancreatic glucagon signals postprandial satiety. Neurosci Biobehav Rev 1990;14:323-338.

30. Werther GA, Hogg A, Oldfield BJ, McKinley MJ, Figdor R, Allen AM, et al. Localization and characterization of insulin receptors in rat brain and pituitary gland using in vitro autoradiography and computerized densitometry. Endocrinology 1987;121:1562-1570.

31. Sieghart W, Sperk G. Subunit composition, distribution and function of GABA(A) receptor subtypes. Curr Top Med Chem 2002;2:795-816.

32. Baez-Mendoza R, Schultz W. The role of the striatum in social behavior. Front Neurosci 2013;7:233. 